\title{
BACTERIOLOGICAL EXAMINATION IN LEPROSY (ITS TECHNIQUE AND INTERPRETATION)
}

K. Ramanujam.

(Reprinted from Laboratory Technicians Bulletin, Vol. II, No. 2, April, 1949.)

The normal habitat of the Mycobacterium lepræ in the human host is the skin, the nasal mucous membrane and the nerve tissue. Depending upon the type of leprosy one is dealing with, it is possible to demonstrate the causative organism of leprosy in one or other of the three situations mentioned. Briefly, the smears taken from the skin and mucous membrane of the nose show a large number of the 
bacilli in cases of infective leprosy, while the scrapings taken from the sheath of the peripheral nerves (ulnars and peroneals) in neural cases very often show a few organisms. The necessity for taking these smears may be either for the purpose of confirming the diagnosis (diagnosis in the first instance being made only on clinical examination) or when one has to assess the progress made by an infective case of leprosy under treatment.

The Methods.

Different methods have been in use for demonstrating the presence of M. lepræ. All of these except one have gone out of vogue for various reasons. However, these methods will be mentioned in passing while the method generally adopted at present will be described in more detail:

I. The 'snip' method: Here a piece of the affected skin is snipped off. This tissue is then rubbed vigorously on the slide until all tissue material is transferred to the slide. The smear is fixed, stained and examined.

2. The 'puncture' method: The skin is pinched hard between the blades of a curved clamp so as to render that part of the skin ischæmic. The skin is then punctured with a thick hypodermic needle. The serum which exudes from the puncture holes is spread on a slide, stained and examined.

3. The 'cutting punch' method: This method consists of removing a piece of skin with a biopsy forceps and smearing the deeper part of the tissue on a slide. The smear is then stained and examined.

4. The 'incision' method:

\section{The Technique of taking Smears by the 'Incision' Method}

Since the Mycobacterium lepræ resides in the layers of the dermis, this method aims at taking the material for examination from that site. This was first outlined by Wade in 1935 and is now widely adopted.

The area to be examined is thoroughly cleansed with cotton wool and spirit and allowed to dry. The skin is pinched up between the thumb and index finger of the left hand and firm pressure applied so as to render the area less vascular. When this is not possible, apply as much pressure as possible laterally. The object of rendering the area ischæmic is to obtain as much of the tissue juice as possible, and the minimum amount of blood which would otherwise make the smear reading difficult.

With a sharp sterile scalpel a small incision is made in the skin (about $5 \mathrm{~mm}$ long), deep enough (about $2 \mathrm{~mm}$ ) to get into the corium. If any blood oozes from the incision it is wiped off. Now 
with the knife blade turned transversely to the line of incision and still retaining the pressure on the fold of the skin with the fingers, the bottom and sides of the incision are scraped repeatedly to obtain a little tissue juice. The material so obtained on the tip of the scalpel is transferred on to a clean microscope slide and a uniform smear is made over a small area. The smear is then fixed by waving it over a flame and stained in the usual way.

There are certain precautions to be taken if one is to guard against errors that might creep in.

(i) The skin should be rubbed vigorously with alcohol, ether or denatured spirit, not only to render it aseptic but also to get rid of any acid fast saprophytes which reside on the skin surface.

(ii) It is necessary to clean the blade of the scalpel with spirit and flame it before using it for taking the next smear, even if it be in the same patient. It is possible that if these precautions are not taken, the bacilli obtained from the first smear may be transferred on to the second one and thereby vitiate the bacteriological readings.

\section{Taking Nasal Smears}

Examination of the scrapings from the nasal mucous membrane forms part of a routine bacteriological examination. The material is obtained by scraping the mucous membrane over the nasal septum well above the external nares.

A sharp pointed tenotomy knife may be used for this purpose. When this is not available a simple cheap instrument suggested by Wade may be used instead. This consists of a paper clip straightened out and one end flattened to form a small scoop. This is then nounted on a piece of wood or bamboo which serves as a handle.

In sufficiently good light the tip of the nose is pushed up with the thumb of the left hand so as to expose the nasal septum. One of these instruments is introduced into the nasal orifice high enough and the mucous membrane covering the nasal septum is scraped off. The material thus obtained is then spread over a clean glass slide so as to give a small uniform smear.

\section{Routine Smears}

Since there is no yardstick by which one can assess the progress made by infective cases of leprosy, we are completely dependent upon the results of smear examination made from time to time. The need for taking multiple skin smears including those from the nose cannot be sufficiently stressed when one realises the "treacherous" nature of the skin lesions in lepromatous leprosy. Areas of skin which appear apparently normal clinically have revealed the presence of $M$. lepræ. However, if this examination is done only for the purpose of confirming the diagnosis, a few 
smears may suffice. But when one has to follow up the cases under treatment, multiple smears are absolutely necessary. It may be said that this practice of taking smears is an infliction on the patient.

It is our practice here to take sixteen smears from each caseone from each ear, cheek, forehead, nose, chest, back, buttock and thigh and also from the chin and arm. It is convenient and also economical to take 8 to Io smears on each slide. In order to avoid any confusion that may arise while the smears are examined, a good practice will be first to make a list of smears to be taken on a piece of paper and then start taking the smears in that order.

\section{Staining of Smears}

\section{Carbol Fuchsin Solution}

Boil Io gms. of Basic Fuchsin in Ioo c.c. of absolute alcohol under a reflux condenser for half an hour. Allow the solution to cool.

Dissolve 50 gms. of Phenol in 950 c.c. of distilled water. Add this to the Fuchsin solution and shake well. Keep for five days for ripening the stain. Filter the solution before use. The solution keeps indefinitely.

\section{(B) The Staining Technique*}

(i) Fix the smears by waving the slide over a flame.

(ii) Flood the slide containing the smears with the 'staining' solution and leave it aside for I5-20 minutes. If there are a number of slides to be stained they may be immersed in a staining jar containing the staining solution. Wash well in running water.

(iii) Decolourise with $15 \%$ sulphuric acid for a few seconds till the smears show a faint pink colour. Wash well in running water.

(iv) Counterstain with $\mathrm{I} \%$ aqueous methylene blue for 3 to 4 minutes. Again wash in running water, allow it to dry, and examine under an oil immersion lens.

While examining smears it is well to bear these things in mind:

(i) When in doubt, the organism is probably not M. leprae.

(ii) At least 200 fields should be examined before a slide is passed as negative.

\& (iii) No slide should be declared positive on the strength of finding a solitary bacillus.

* This method has been modified as described in the original article. -EDITOR. 
While examining the smears one will encounter the organisms in various stages of disintegration. They may appear beaded, fragmented and granular, and sometimes as acid-fast dust. It will also be advantageous to make a note of the nature of the organism seen-(e.g., Rt. ear $4+$ fragmented; Lt. ear $3+$ granular; Rt. cheek $5+$, etc.)

\section{Conclusions}

When taking material for bacteriological examination it is well to remember the following points:-

(I) Have the patient in good sunlight and take smears from the obviously affected or suspicious areas. Take as many as 16 smears as a routine from the areas mentioned earlier.

(2) Take as much of the tissue pulp and as little blood as possible so as to render the microscopic examination easy and clear.

(3) Always make it a point to flame and clean the blade of the knife between successive smears so as to avoid errors.

(4) When in doubt about the identity of the organism never call it M. lepræ.

I wish to acknowledge my grateful thanks to Dr. R. G. Cochrane for permitting me to write this paper and for his valuable suggestions. References:

(I) Cochrane, R. G.-A Practical Text Book of Leprosy (1947).

(2) Muir, Ernest-Manual of Leprosy (1948).

(3) Muir, E.-Leprosy; Diagnosis, Treatment and Prevention

(6th edition).

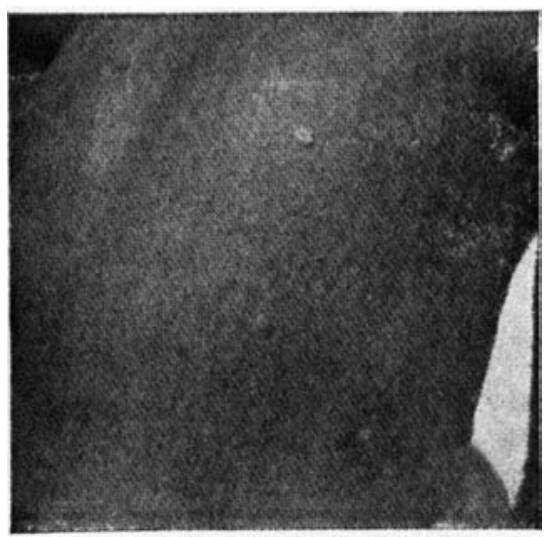

The lesion to be sueared

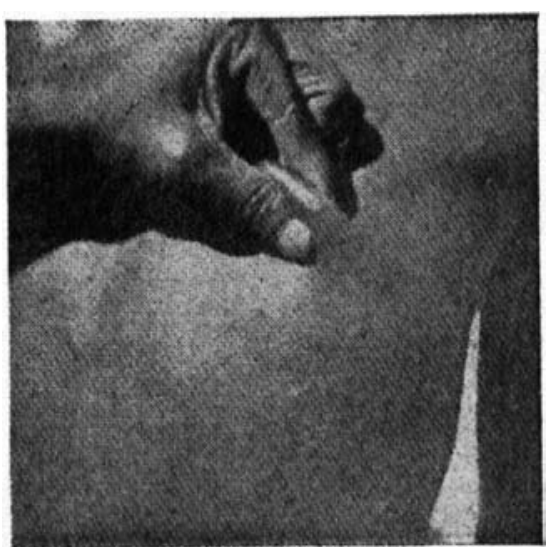

The lesion pinched up in a fold of the skin 


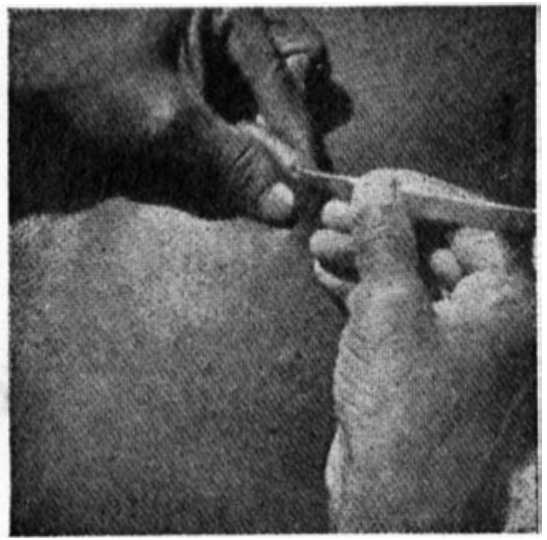

i The incision made

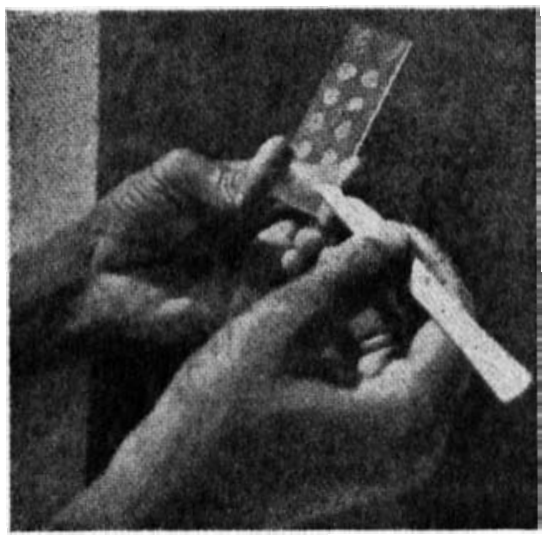

The material transferred to a clean slide so as to make a thin small smear

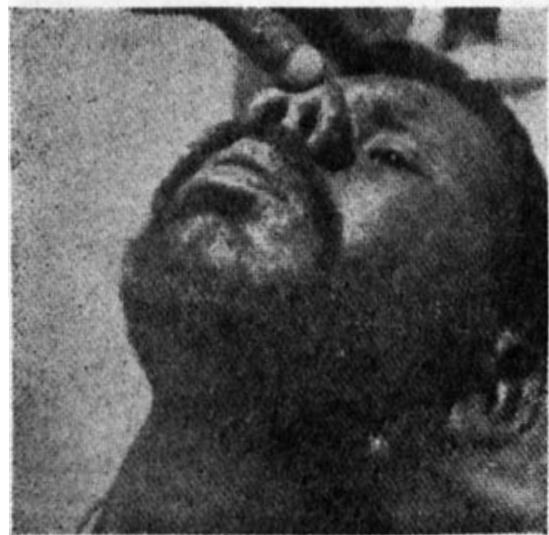

Taking Nasal Smear The tip of the nose is pushed up

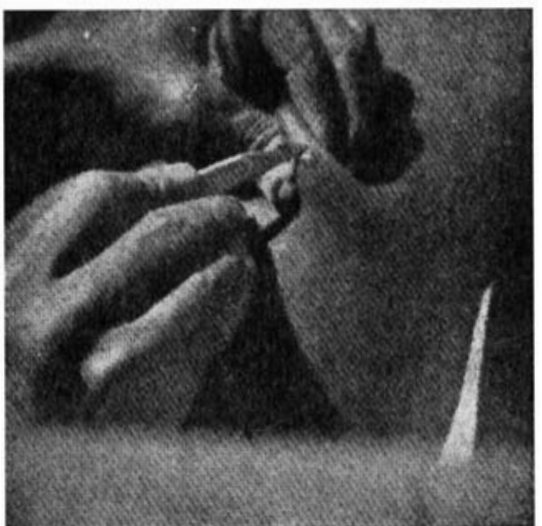

T'he tissue pulp scraped

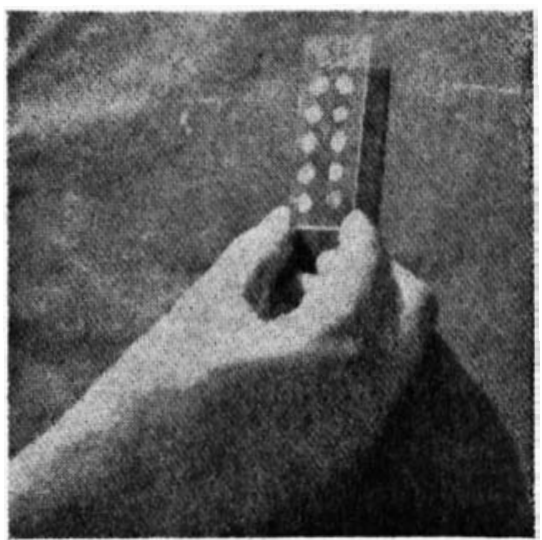

The slide with multiple smear ready for staining

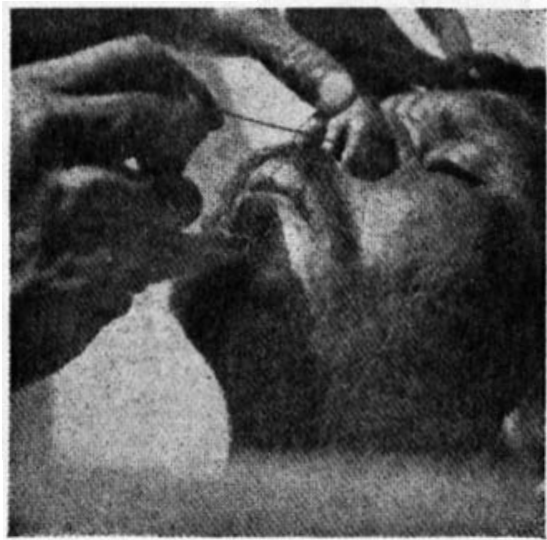

Taking Nasal Smear

With a suitable instrument the imucous membrane over the nasal septum is scraped 